\title{
Experimental study of passive compensation of space charge at the Los Alamos National Laboratory Proton Storage Ring
}

\author{
M. A. Plum, D. H. Fitzgerald, J. Langenbrunner, R. J. Macek, F. E. Merrill, F. Neri, H. A. Thiessen, and P. L. Walstrom \\ Los Alamos National Laboratory, P.O. Box 1663, Los Alamos, New Mexico 87545 \\ J.E. Griffin,* K. Y. Ng, Z.B. Qian, and D. Wildman \\ Fermi National Accelerator Laboratory, P.O. Box 500, Batavia, Illinois 60510 \\ B. A. Prichard, Jr. \\ SAIC, 122 Longview Drive, Los Alamos, New Mexico 87544
}

(Received 5 February 1999; published 29 June 1999)

\begin{abstract}
The inductance of the vacuum chamber of the Proton Storage Ring at Los Alamos National Laboratory was intentionally increased by the introduction of ferrite rings to counteract the longitudinal space-charge effect of the intense beam. The magnetic permeability of the ferrite could be adjusted by introducing current into solenoids wound around the ferrite. Results show that the minimum rf voltage necessary to stabilize the beam against $e-p$ instability may be reduced over that previously measured. The injected bunch length was observed to be longer when the ferrite was heavily biased so that its effect was reduced. [S1098-4402(99)00048-8]
\end{abstract}

PACS numbers: 29.20.Dh, 29.27.Bd

\section{INTRODUCTION}

Several relatively small $(\sim 100-1000 \mathrm{~m}$ circumference) proton rings, to be operated at very high intensity $\left(10^{14}\right.$ protons per cycle), are presently being proposed as integral parts of programs such as neutron spallation sources or meson/hadron facilities [1-4]. $\mathrm{H}^{-}$ions are to be injected into the rings for many turns at relatively low energy $(\sim 1 \mathrm{GeV})$. In the spallation sources, gated linac pulses are to be injected into stationary rf buckets at the rotation frequency $(h=1)$ in order to preserve an extraction gap, as well as to allow electrons to escape from the potential well created by the stored protons. In the hadron/meson sources, such rings are to be components of a series of rapid cycling synchrotrons. Some form of $\mathrm{rf}$ capture (quasi-adiabatic capture or possibly longitudinal painting into preset rf buckets) at low harmonic number is anticipated.

In each case, longitudinal space-charge fields can generate substantial distortion of the rf-generated potential wells. The effective space-charge voltage acting on a particle is proportional to the slope of the line charge distribution $\lambda(s)$, where $s$ is the distance coordinate along the particle closed orbit [5-7]. Below transition energy, the space-charge forces counteract the effectiveness of rfgenerated bunching forces.

The space-charge reduction in the rf-generated bucket area can be compensated to first order by increasing the rf voltage at the chosen harmonic. But even with increased rf voltage, the space-charge fields distort the bucket shape,

*Retired. affect the incoherent synchrotron tune spread, and have the potential for causing instability and longitudinal emittance growth. In the meson source case, it is planned to reduce the bunch length to the minimum possible value prior to extraction. Longitudinal emittance dilution during acceleration will adversely affect the final bunch length. For proton storage rings, the space-charge force may significantly reduce the effectiveness of the rf system in preserving an adequate gap in the beam.

The major Fourier components of the beam current in such machines will be in the $\mathrm{MHz}$ range. At these frequencies, the vacuum-chamber impedance will be predominantly inductive. The wall image of the beam current will generate an effective voltage which is proportional to the slope of the line charge distribution. Below transition, the phase of the beam induced voltage is such that it will induce self-bunching of the beam. This implies that the vacuum-chamber induced voltage will strengthen the longitudinal focusing force developed by the externally applied rf. The net effective voltage per turn resulting from ring inductive wall impedance and the space-charge selfvoltage can be expressed:

$$
V_{s}=\frac{\partial \lambda(s)}{\partial s}\left[\frac{g_{0} Z_{0}}{2 \beta \gamma^{2}}-\omega_{0} L\right] e \beta c \bar{R}
$$

or

$$
\begin{aligned}
V_{s}=2 I_{\mathrm{dc}} \frac{d}{d t} \sum_{m=1}^{\infty} & F_{n}\left(m \omega_{\mathrm{rf}}\right) \cos \left(m \omega_{\mathrm{rf}} t\right) \\
& \times\left[\frac{g_{0} Z_{0}}{2 \beta \gamma^{2} \omega_{0}}-L(\omega)\right] .
\end{aligned}
$$

In these expressions, $I_{\mathrm{dc}}$ is the $\mathrm{dc}$ beam current, $F_{n}\left(m \omega_{\mathrm{rf}}\right)$ are normalized Fourier components of the 
beam current distribution $e \beta c \lambda(s)$ at harmonics $n$ of the rotation angular frequency $\omega_{0}, \omega_{0} L$ is the vacuumchamber inductive reactance (nominally 15-25 $\Omega$ ) at the beam angular rotation frequency and is referred to as the inductive part of $Z_{\|} / n, \beta$ and $\gamma$ are relativistic parameters, $e$ is the electron charge, $\bar{R}$ is the average machine radius, and $c$ is the velocity of light. $Z_{0} \approx$ $377 \Omega$ is the impedance of free space and $g_{0}=1+$ $2 \ln (b / a)$ is the geometric space-charge constant, where $a$ and $b$ are the nominal beam radii and vacuum-chamber aperture [8]. This form of $g_{0}$ is derived by calculating the space-charge potential on the beam axis for a uniform circular beam in a round vacuum chamber. Beams in the machines under consideration will almost certainly not meet these specifications; therefore, the constant may be imprecisely known, ranging from $\sim 2$ to $\sim 3$. The space-charge part of $Z_{\|} / n$ in Eq. (1.1) falls in the range $100-250 \Omega$.

In Eq. (1.1), $L$ is usually assumed to be independent of frequency. The signs in the equations are consistent with the sign of the applied rf voltage below transition. In Eq. (1.2), the expression $L(\omega)$ implies that the vacuumchamber inductance may be a function of frequency, particularly if the vacuum-chamber inductance is augmented by the introduction of ferrite, as will be described in this paper. By adjusting the value of $L(\omega)$, it is apparent that, at some chosen energy, the term in brackets and, consequently, the space-charge effect may be substantially reduced or canceled. This concept was proposed in 1968 by Sessler and Vaccaro [9] and in 1982 by Cooper et al. [10] and Hardek [11].

In 1966 Neil and Briggs also proposed intentional insertion of inductance into particle beam vacuum chambers [12,13]. This was intended as a mechanism to oppose space-charge self-bunching above transition and, thereby, to stabilize ion beams against negative-mass or microwave instability. In the experiment to be considered here, the beam energy will be below transition energy.

\section{LANL PROTON STORAGE RING}

The Proton Storage Ring (PSR) at Los Alamos National Laboratory (LANL) has a circumference of $90.2 \mathrm{~m}$ and is filled by multi-turn $\mathrm{H}^{-}$injection from the Los Alamos Neutron Science Center (LANSCE) linac at $797 \mathrm{MeV}$. The transition gamma is $\gamma_{t}=3.1$. We therefore have revolution frequency $f_{0}=2.796 \mathrm{MHz}$, period $357.7 \mathrm{~ns}$, $\beta=0.8412$, and slip factor $\eta=-0.1883$.

The rms injected momentum spread is $\Delta p / p \approx$ $0.063 \%(0.77 \mathrm{MeV})$. The $\sim 10 \mathrm{~mA}$ linac pulses are gated during normal operation to $\sim 250 \mathrm{~ns}$, and the rotation period is synchronized to the injection rate such that injected pulses are superposed, initially allowing a gap of about $108 \mathrm{~ns}$ in the beam. Injection for 1600 turns, requiring $572 \mu \mathrm{s}$, fills the ring with roughly $2.5 \times 10^{13}$ protons. If nothing is done to prevent it, protons will drift into the gap during injection due to space-charge forces, natural azimuthal motion due to the slip factor $\eta$, and the injected momentum spread.

Depending on the rf voltage, the PSR suffers from a serious instability for beam intensities greater than $\sim 1 \times$ $10^{13}$ stored protons, which limits operation at higher intensities because of transverse beam loss [14-16]. Strong evidence exists that the instability is caused by electrons trapped in the proton-beam potential well $[17,18]$. Preservation of a proton-free gap of about $100 \mathrm{~ns}$ in the beam (required for extraction kicker clearance in any case) has been shown to increase the threshold for the putative $e-p$ instability. The required gap is presently maintained with an installed $h=1(2.796 \mathrm{MHz})$ rf cavity [11] capable of operating at $\sim 12 \mathrm{kV}$. Evidence for improving the threshold of the intensity-limiting instability by raising the $\mathrm{rf}$ voltage is shown in Fig. 1.

At the start of injection, the nominal operating rf voltage is set at $2 \mathrm{kV}$ and is increased gradually to about $7 \mathrm{kV}$. The bucket height changes from $\sim 3$ to $\sim 5.4 \mathrm{MeV}$, and the small-amplitude synchrotron period decreases from 1.6 to $0.87 \mathrm{~ms}$. With this voltage program, it appears that some fraction of the injected beam falls outside of the (cosine contour) bucket separatrices. Such particles will move on orbits that will carry them into the gap region, where only a small fraction of the injected beam is sufficient to prevent the escape of the captured electrons. Protons injected within the separatrices in the injection process complete less than one phase oscillation before extraction. If the $h=1 \mathrm{rf}$ voltage is increased substantially in order to capture more of the injected beam, some orbits may carry particles beyond the momentum aperture of the ring, causing beam loss.

As part of a major upgrade in the PSR beam delivery capability [19], there is a multi-stage program to upgrade the ring rf system. The fraction of the beam captured within the synchrotron separatrices could be increased

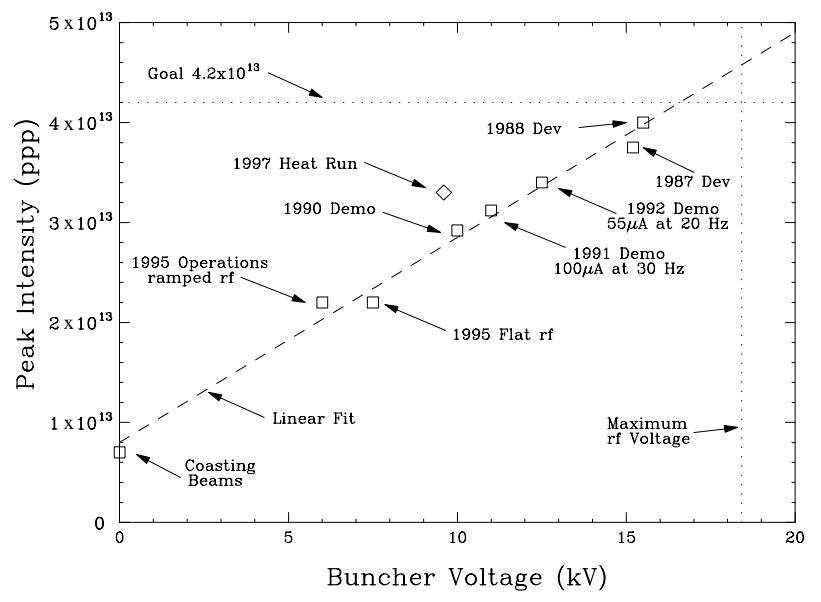

FIG. 1. Maximum stable stored beam intensity as a function of rf bunching voltage. 
without a large increase in the total momentum spread by adding harmonics to the $h=1 \mathrm{rf}$ voltage. The added harmonics would reduce the momentum excursion of the orbits near the center of the phase space. To this end, it has been proposed to increase the $h=1$ voltage capability to $18 \mathrm{kV}$ and to add $15 \mathrm{kV}$ and $4 \mathrm{kV}$ capability at the second and third harmonics, respectively. This, in effect, will create a longitudinal barrier [20] by appropriate phasing of the additional Fourier components. In addition to these measures, it has been proposed [21] that the performance of the upgraded rf system be augmented by the installation of passive broadband spacecharge compensating inductors.

\section{SPONTANEOUS SELF-BUNCHING}

The effect of moderately large real or reactive vacuumchamber impedance on the PSR beam has been observed in a recent PSR experiment. The beam was injected into the ring with the rf power amplifier disabled and the cavity tuned below, near, and above resonance. In normal operation, each side of the rf-cavity accelerating gap is driven by a cathode-follower amplifier with a driving point impedance of $\sim 20 \Omega$, so that the impedance presented to the beam is very low and quite insensitive to cavity tuning.

In a LANL experiment performed in February 1997, rf voltage was developed at the cavity gap by beam excitation alone. The power amplifier system was turned off and the intermediate power amplifiers were disconnected from the grids of the final amplifier tubes. The cathodes of the final amplifier tubes were shorted to the grids to prevent beam excitation of the cavity impedance from developing excessive grid-to-cathode voltage. Using reasonable estimates for the beam current and momentum spread during the experiment, the threshold for inductive-wall "instability" can be set between $\operatorname{Im} Z_{\|} / n=100$ and $200 \Omega$.

The amplitude of the $\mathrm{rf}$ signal developed by the 2.8 $\mathrm{MHz}$ Fourier component of the beam current on the buncher rf-cavity gap was recorded during filling and storage for each of the tuning conditions. Plots of the voltages are shown in Fig. 2. The figure shows the rf voltage developed at the cavity by the $2.8 \mathrm{MHz}$ Fourier component of the beam. Larger signals imply shorter bunches while smaller signals imply longer bunches. In this experiment, the injected beam bunch width was about $200 \mathrm{~ns}$, somewhat shorter than the 250 ns normally used. The current stored in the PSR was limited by injecting every other turn to protect the output tubes. The total beam stored in the PSR was approximately $10^{13}$ protons per pulse under these conditions. Data were taken at three conditions of the cavity tuning bias: $280 \mathrm{~A}$ (resonant frequency $2.8 \mathrm{MHz}$ ), $250 \mathrm{~A}$ (resonant frequency $2.68 \mathrm{MHz}$ ), and $311 \mathrm{~A}$ (resonant frequency $2.95 \mathrm{MHz}$ ). In these three cases, the cavity presented real, capacitive, and inductive reactance, respectively, to the beam.

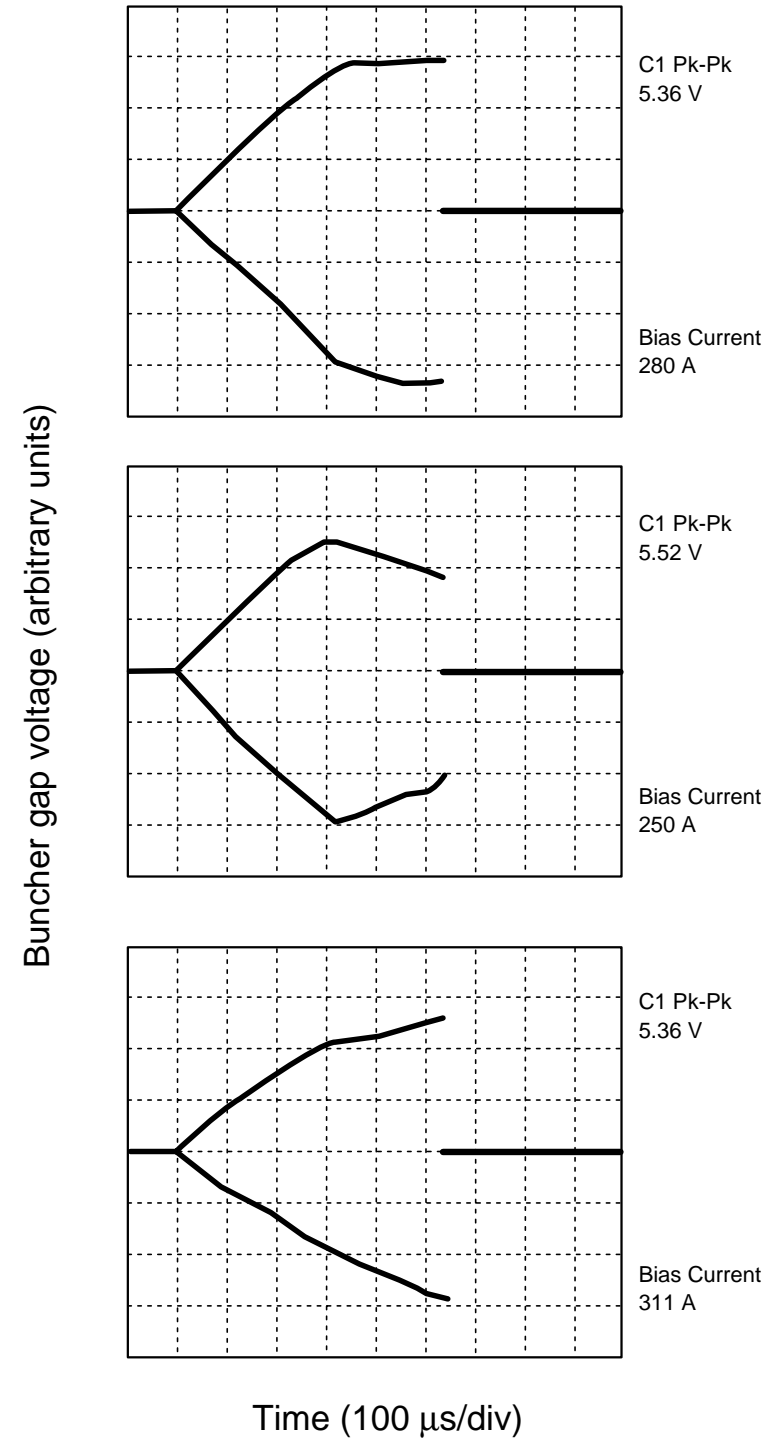

FIG. 2. Beam induced rf voltage on the LANL PSR $2.8 \mathrm{MHz}$ cavity during the injection and storage of the beam. The envelope of the induced rf voltage is shown in arbitrary units, the time scale is $100 \mu \mathrm{s}$ per division. The start of injection is at about $80 \mu \mathrm{s}$, the end of injection occurs at approximately $400 \mu \mathrm{s}$, and extraction occurs at approximately $620 \mu \mathrm{s}$ (accounting for the rapid drop in $\mathrm{rf}$ voltage).

The oscilloscope traces recorded in the latter two cases show that the beam debunches if the cavity is tuned to a lower frequency than the beam (capacitive) and that the beam self-bunches if the cavity is tuned to a higher frequency than the beam (inductive). Note that the rise time of the $2.8 \mathrm{MHz}$ component of the self-bunching (debunching) cavity voltage is long compared with the storage time of the PSR, in this case approximately $530 \mu \mathrm{s}$. Also note that in normal operation of the PSR, the low output impedance of the cathode-follower drive completely overcomes this phenomenon, and no such bunching or debunching is seen. 


\section{STABILITY CONSIDERATIONS}

Below transition, effective ring voltages developed by Fourier components of beam current in the vacuumchamber inductance are of such a phase as to bunch some fraction of the beam. If the ring inductive reactance at some frequency $n f_{0}$ is sufficiently large so that a beam Fourier component at that frequency can generate a ring voltage large enough to generate buckets that contain the entire beam longitudinal emittance, then the ring impedance has exceeded the Keil-Schnell instability threshold [22]. This is essentially an instability situation, which may be stabilized to some extent by counteracting space-charge forces.

By examining the growth rate for the longitudinal resistive-wall instability in the PSR (after the proposed upgrades), an acceptable longitudinal impedance has been calculated by Channell and Wang [23] to be $\operatorname{Re} Z_{\|} / n \leq$ $1 \mathrm{k} \Omega$ and $\left|\operatorname{Im} Z_{\|} / n\right| \leq 10 \mathrm{k} \Omega$. The value of $g_{0}$ has been reported [24] to be $\sim 3$. Recent increases in the vertical beam size may have reduced this value to near 2.5. Depending on the choice of $g_{0}$, the space-charge impedance per harmonic is $\sim 160-200 \Omega$ at $2.8 \mathrm{MHz}$. Introduction of inductive reactance of this magnitude, if approximately compensated for by the space-charge term, should not exceed the instability threshold. But, in any case, the controlled spontaneous self-bunching would present a problem only if it resulted in a momentum spread sufficiently large to exceed the ring momentum aperture during the short storage time.

Introduction of a ferromagnetic liner with the same beam clearance diameter as the original vacuum chamber has negligible effect on the beam-to-wall capacitance per unit length. The ferrite material has relative electric permittivity $\epsilon_{r} \sim 9$, so very little electric field energy is stored within the ferrite. In some sense, it amounts to placing a large capacitance in series with a small one. The net capacitance is not changed by a significant amount. An analysis of the wake fields developed by a relativistic particle beam in a dielectric-lined waveguide has been presented by $\mathrm{Ng}$ [25]. Based on that work, a detailed analysis of the stability conditions associated with introduction of an inductive region (ferrite waveguide) into the vacuum chamber by coating the inner wall of the chamber with ferrite has been completed by $\mathrm{Ng}$ and Qian [26,27].

The major sources of longitudinal resistive impedance have been calculated [28] to be the extraction kickers $\left(\operatorname{Re} Z_{\|} / n \approx 20 \Omega\right)$ and the $\mathrm{rf}$ cavity $\left(\operatorname{Re} Z_{\|} / n \approx 20 \Omega\right)$. Other impedance sources include the stripline beam position monitors and the clearing electrodes, but these impedances should be relatively small. A rough estimate of the total impedance of the PSR before adding the inductor is $\operatorname{Re} Z_{\|} / n<100 \Omega$ and $\left|\operatorname{Im} Z_{\|} / n\right|<300 \Omega$. Measurements (described in the appendix) of the resistive part of the inductor impedance give $\operatorname{Re} Z_{\|} / n=0.46 \Omega$, so the resistive effect of adding the proposed inductance to the
PSR is insignificant. The total resistive impedance per harmonic remains below the $1 \mathrm{k} \Omega$ limit.

The probability that new impedance-related instabilities will be driven by the installation of space-charge compensating inductance is expected to be minimal.

\section{PROPOSED EXPERIMENT}

The space-charge compensation under consideration differs from the introduction of inductive reactance at the beam fundamental $\mathrm{rf}$ frequency (i.e., a detuned resonant cavity) in that it is proposed to insert untuned inductance which is to remain reasonably constant over a large frequency range, spanning as many rotational harmonics as possible. Operation of such a passive compensation system has not been documented in a high-current storage ring. The possibility that such a system may not work as planned and that it may introduce unanticipated instabilities should not be ignored. Therefore, as a test of the feasibility, stability, and efficacy of such a system, a shortterm ad hoc collaboration between LANL and Fermilab was formed. Fermilab fabricated and measured two ferrite waveguides with total inductance equal to approximately $60 \%$ of that believed to be necessary to compensate for the expected space-charge impedance generated by the PSR beam. The effect of the installation should be observable while minimizing the risk of unanticipated instability problems. Just before a PSR shutdown period related to the LANSCE Reliability Improvement Project upgrade, the inductors were installed in the PSR. Several shifts of beam study were allocated to understanding the performance of the added inductance.

At the PSR rotation frequency, $2.8 \mathrm{MHz}$, the requisite space-charge compensation impedance per harmonic, $\sim j 200 \Omega$, would require installation of $\sim 11 \mu \mathrm{H}$ inductance. At Fermilab there is a supply of ferrite rings (Toshiba $\mathrm{M}_{4} \mathrm{C}_{21 A}, 12.7 \mathrm{~cm}$ i.d., $20.3 \mathrm{~cm}$ o.d., and $2.54 \mathrm{~cm}$ thick). The relative magnetic permeability is 50 to 70 (over a modest temperature range) with $Q \sim 150$. These properties remain approximately constant up to $30 \mathrm{MHz}$. The properties of the ferrite are not expected to be adversely affected by the dc or rf magnetic fields generated within the ferrite by the PSR beam. (Detailed measurements of the ferrite properties are discussed in the appendix.)

A ferrite waveguide composed of a stack of these cores should have inductance roughly $4.7 \mu \mathrm{H} / \mathrm{m}$. Complete compensation would require $234 \mathrm{~cm}$ (92 in.), or 92 1-in.-cores. Two vacuum-tight welded stainless-steel containers were prepared, each containing $75.5 \mathrm{~cm}$ of continuous ferrite. (It was intended to insert 30 cores in each container, but due to a machining error the chambers were $0.76 \mathrm{~cm}$ too short and one core in each chamber was ground down to fit.) Each container has $12.7-\mathrm{cm}-$ i.d. stainless-steel tubes terminated in vacuum flanges at each end. To prevent charge buildup on the inner surface of the cores, each of the cores was treated with a very thin 
(1 $\mathrm{M} \Omega$ per square) conductive coating (Heraeus R8261) baked on the inner and outer surface. Additional radial conducting "spokes" were added to provide conductivity from the inner surface to the outer wall of the chamber.

The energy delivered to the ferrite at $2.8 \mathrm{MHz}$ due to the fundamental Fourier component of the beam current (clearly the largest component) is negligible. Assuming that the $2.8 \mathrm{MHz}$ component of beam current is $20 \mathrm{~A}$, the ferrite magnetic stored energy is $W_{\mathrm{avg}}=L I^{2} / 4=4.2 \times$ $10^{-4} \mathrm{~J} / \mathrm{m}$. Assuming $Q=100$, the power dissipation is $\sim 74 \mathrm{~W} / \mathrm{m}$, or $\sim 107 \mathrm{~W}$ total. This amounts to a decelerating voltage of $\sim 5 \mathrm{~V}$ per turn. The sum of the power delivered to the ferrite in all of the remaining harmonics, even with deteriorating $Q$ above $50 \mathrm{MHz}$, probably will not exceed this value.

To provide a variable "knob" so that the effective inserted inductance could be varied during the experiment, a solenoid, made from 88 turns of welding cable, was wrapped around the outside of each tank. Experience with the same ferrite in a similar geometry, effectively a Fermilab-style rf-cavity tuner geometry, showed that the relative permeability could be reduced by more than a factor of 2 by applying $10^{5}$ A turns/m "orthogonal" $\mathrm{dc}$ bias [29]. The two inductor tanks were installed adjacent to each other in the PSR enclosure. The ferrite bias windings were connected in series, and the sense of the current in the windings was set so that the stray magnetic fields on the beam axis would oppose each other. Each of the tanks was provided with a steel magnetic-field return path to minimize stray fields. Safety considerations dictated that each of the coils could be energized periodically to a level of $1000 \mathrm{~A}$ for periods no longer than one minute.

\section{EXPERIMENTAL RESULTS}

Two $12 \mathrm{~h}$ shifts on August 2 and 3, 1997, were allocated to the study of passive compensation in the PSR. The first goal of the experiment was to ascertain that the installation of the inductors did not adversely affect normal operation or beam losses in the PSR or introduce any new instabilities. The second goal was to establish that the inductors did indeed interact with the beam as broadband inductors and to determine the degree to which the installation counteracts the space-charge forces. The third goal was to make a comparative measurement of the $e-p$ instability threshold with and without the solenoid bias at several beam intensities. Here it is assumed that the PSR instability is, in fact, caused by electrons and that reducing the number of protons in the rf-generated gap will increase the instability threshold.

\section{A. Normal operation}

Normal operation of the PSR was established without incident. It was determined that the presence of the inductors, with and without bias, did not affect the previously established PSR magnet settings or closed orbit. Measurements of the first turn, closed orbit, fractional betatron tunes, extracted beam profiles, ring beam loss pattern, and extraction beam-line losses indicated no change with the solenoids off or on at 200 A. This observation corroborated calculations of the possible betatron tune shifts which might be caused by linear focusing effects of solenoids. No new or unanticipated instabilities were observed.

\section{B. Space-charge compensation}

To study space-charge compensation caused by the installed inductance, two experiments, using different bunch lengths, were completed. The designated charge configurations were injected into the PSR and the longitudinal profiles (bunch length and shape) were observed, digitized, and recorded using signals from a wide-band wall current monitor. The pulse-shape data were recorded at the end of each $625 \mu$ s injection period. The resulting waveforms were compared with detailed particle tracking simulations.

The rf voltage was set to $7 \mathrm{kV}$ and the injected bunch lengths, termed pattern widths, were 50 and $150 \mathrm{~ns}$. In each case, the beam was injected for $625 \mu \mathrm{s}$. The absolute number of particles in the injected beam was obtained from the normalization of the PSR current monitor SR-CM42. (The calibration is approximately $2.2 \times 10^{13}$ protons per volt). At the end of injection for the $50 \mathrm{~ns}$ pattern width, the number of protons was about $4 \times 10^{12}$. For the $150 \mathrm{~ns}$ pattern-width experiment, the proton number was about $1.2 \times 10^{13}$. The absolute normalization of the current monitor is not better than about $10 \%$. The relative normalization is better if the data are taken in rapid succession, which was done to the extent limited by the time required to apply the biasing current to the solenoids. The total number of particles was the same for the bias-on and bias-off curves with at most a $1 \%$ to $2 \%$ difference. To check for data reproducibility from one injection cycle to the next, the pattern of measurements was bias off, bias on, bias off again, and bias on again. Representative data for the $50 \mathrm{~ns}$ experiment and simulation results reflecting the same operating conditions are shown in Fig. 3.

The simulations were done with an assumed injection momentum spread $\Delta p / p=0.08 \%$, corresponding to an energy spread $0.985 \mathrm{MeV}$. In the simulations, the areas under the curves, representing the total charge, were normalized to the areas of the experimental data curves. The precision of the normalization is $1 \%$ to $2 \%$.

The solid curve in the left plot of Fig. 3 represents the bunch shape with the full effect of the inserted inductance (zero bias). The dotted curve corresponds to data with the effect of the inductance diminished by a $900 \mathrm{~A}$ dc bias. The ratio of peak heights is about $16 \%$. Differences of this magnitude were observed in each of the repetitions of the experiment. Because the injected beam intensity and 

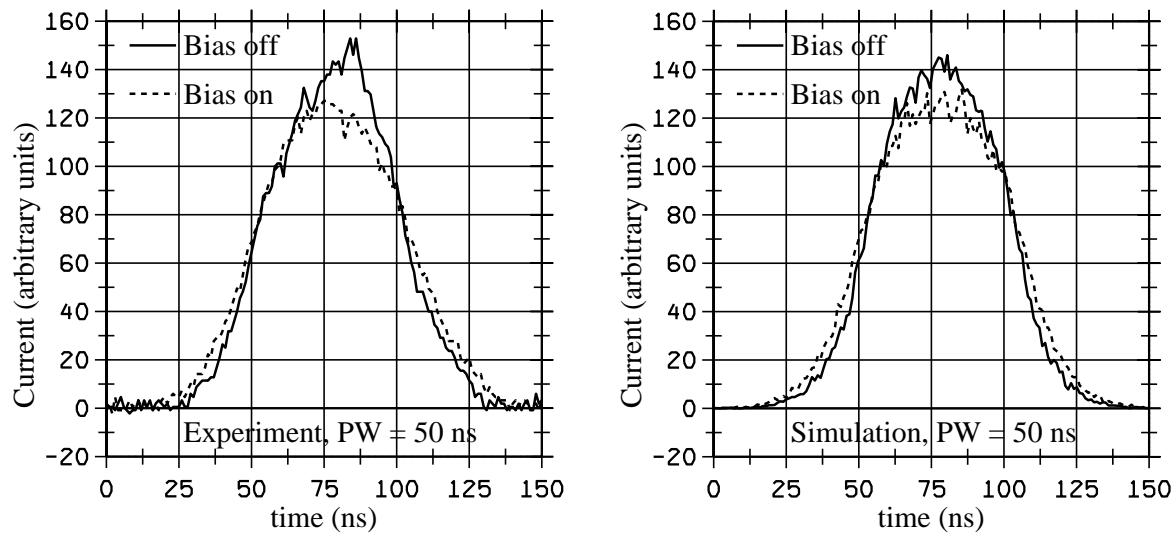

FIG. 3. Measured (left) and simulated (right) pulse shapes after $625 \mu$ s for an injected pattern width of 50 ns. The simulations used $4 \times 10^{12}$ protons. Solid curves represent the shape with no bias on the inductor $(7.29 \mu \mathrm{H}$ in the simulation). Dotted curves represent the shapes with a $900 \mathrm{~A}$ bias applied to the inductor $(2.49 \mu \mathrm{H}$ in the simulation). Buncher voltage was $7.5 \mathrm{kV}$.

pattern widths were held as constant as possible during all repetitions, the differences in pulse amplitude are not likely to be due to fewer particles in the bias-on case. Also, it was clearly evident that the bias-on experimental curves were wider than the bias-off curves in each repetition. The areas under the bias-on and the bias-off data curves were found to agree within $1 \%$ to $2 \%$.

For a total effective inductance of $7.29 \mu \mathrm{H}$, the simulations predict an rms bunch length of $19 \mathrm{~ns}$. Raising the ferrite bias current to $900 \mathrm{~A}$ reduces the inductance to $2.49 \mu \mathrm{H}$, and the simulations predict that this should increase the rms bunch length to $22 \mathrm{~ns}$. The solid data curve in the left plot of Fig. 3 is well matched to a cosinesquared line charge distribution with rms length of $19 \mathrm{~ns}$ and full width at base of $105 \mathrm{~ns}$. These data are consistent with the simulation prediction. The lower-amplitude bias-on dotted curve is not easily measured or matched, but it is not inconsistent with the simulation prediction. In each observation, the bunch amplitude was smaller and the bunch width was broader after application of the ferrite bias. To check that the pulse width sharpening is a reproducible effect, data from several consecutive passes of the bunch around the PSR were examined. The character of the data remained unchanged, as might be expected, because the period of a single turn is an extremely small fraction of a synchrotron phase-oscillation period.

The left and right plots of Fig. 4 show the experimental results and the simulations, respectively, for the $150 \mathrm{~ns}$ pattern-width injection cases. The effect of the bias is less pronounced in this case and masked to some extent by noise. Still, the simulations reproduce the magnitude of the experimental effect within experimental errors. The character of the pulse height and width changes with and without ferrite bias is essentially the same as those for the $50 \mathrm{~ns}$ case. The changes, though small, were observed consistently in all of the data examined.

The agreement between the experimental data and the simulations is reasonably good and the peak-to-peak ratio (about $16 \%$ between the bias-on and bias-off cases) agrees with the experiment within an error of $2 \%$ to $3 \%$. One difference between the experimental data and the simulation is the asymmetry of the experimental curves
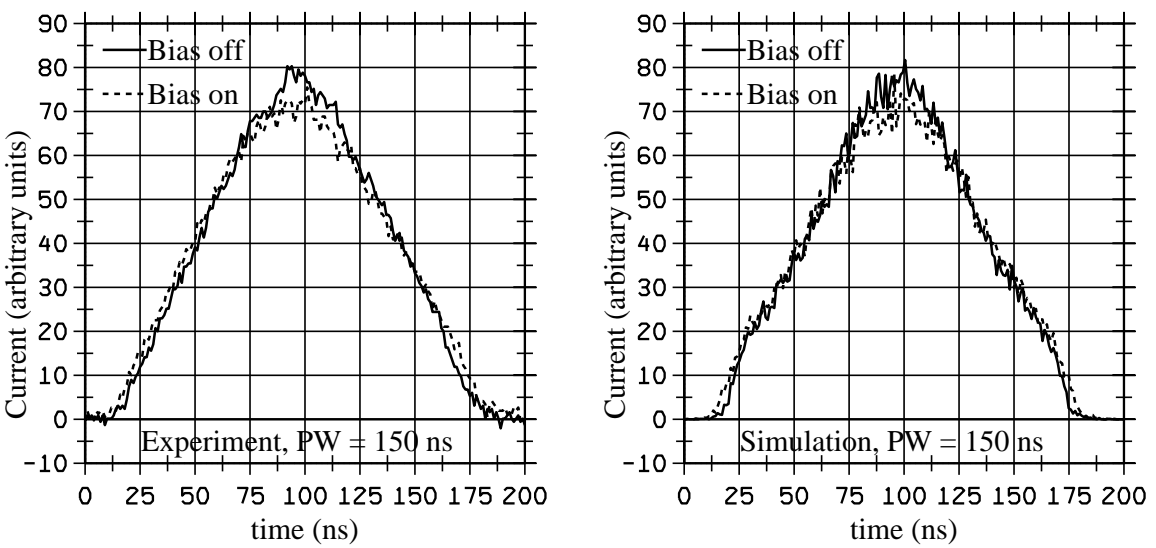

FIG. 4. Measured (left) and simulated (right) pulse shape after $625 \mu$ s, with an injected pattern width of $150 \mathrm{~ns}$. The simulations used $1.2 \times 10^{13}$ protons. Solid curves represent the shape with no bias on the inductor $(7.29 \mu \mathrm{H}$ in the simulation). Dotted curves represent the shapes with a $900 \mathrm{~A}$ bias applied to the inductor $(2.49 \mu \mathrm{H}$ in the simulation). Buncher voltage was $7.5 \mathrm{kV}$. 
in contrast with the much more symmetric results from the simulations. This difference could simply be due to a slight error in the setting of the buncher phase. It could also be due to an asymmetry in the injected beam momentum distribution. The effect of the second order (in $\Delta p / p)$ terms in the time of flight are too small to explain the visible asymmetry. In summary, the simulations are in good agreement with the experimental results in the $50 \mathrm{~ns}$ pattern-width case and not inconsistent in the 150 ns case.

The bunch-length changes observed in this set of experiments, though not dramatically large, support the contention that the introduction of passive inductance into the vacuum chamber will act in a manner similar to that of the rf voltage in creating a potential well capable of containing the longitudinal excursions of a bunched proton beam. If it is assumed that biasing the ferrite reduces the ferrite effectiveness to a negligible level, then the bunch-length reduction resulting from the introduced inductance $(50 \mathrm{~ns}$ case) is about $10 \%$. Clearly the inductance has some effect fully biased, so this is a "worst case" scenario. For a single-frequency sinusoidal rf system and a beam with a defined initial longitudinal emittance, the bunch length scales with rf voltage as $V_{\mathrm{rf}}^{-1 / 4}$. This scaling implies that the rf voltage would have to be raised by a factor larger than $\sim 1.5$ to obtain the same bunch narrowing without the inductance.

\section{Effect on the PSR beam instability}

In an attempt to isolate the effect of the inductors on the instability threshold, a preliminary instability threshold measurement was made at the beginning of the inductor experiment. The $\mathrm{rf}$ was on a ramped profile. The beam conditions were $625 \mu$ s beam gate, $250 \mathrm{~ns}$ pattern width, and $100 \mu$ s storage time. With $2.9 \mu \mathrm{C}$ of charge or $1.8 \times 10^{13}$ protons in the ring, the beam was marginally unstable for a peak buncher voltage of $5.0 \mathrm{kV}$. (Marginally unstable means beam loss close to the end of the $100 \mu \mathrm{s}$ storage time.) The buncher rf-voltage profile was then changed to a flat top (constant during the injection and storage periods), and the rf voltage needed for the marginally stable beam was lowered to $3.645 \mathrm{kV}$. The inductor solenoids were then turned on to $900 \mathrm{~A}$, and the rf voltage needed for the marginally stable beam was lowered to about $3.094 \mathrm{kV}$. These data, added to the historical data of Fig. 1, are shown in Fig. 5 by three triangles connected by a horizontal line.

The flat-top buncher data point is contrary to measurements [30] made before installation of the inductor, where it was observed that the buncher voltage at the instability threshold is $20 \%$ lower if a ramped $\mathrm{rf}$ profile is used. The biased inductor data point is also surprising because it was expected that application of a 900 A ferrite bias would decrease the effectiveness of the inductors and the rf voltage would then have to be increased to reestablish the threshold for instability. Instead, a lower buncher voltage was

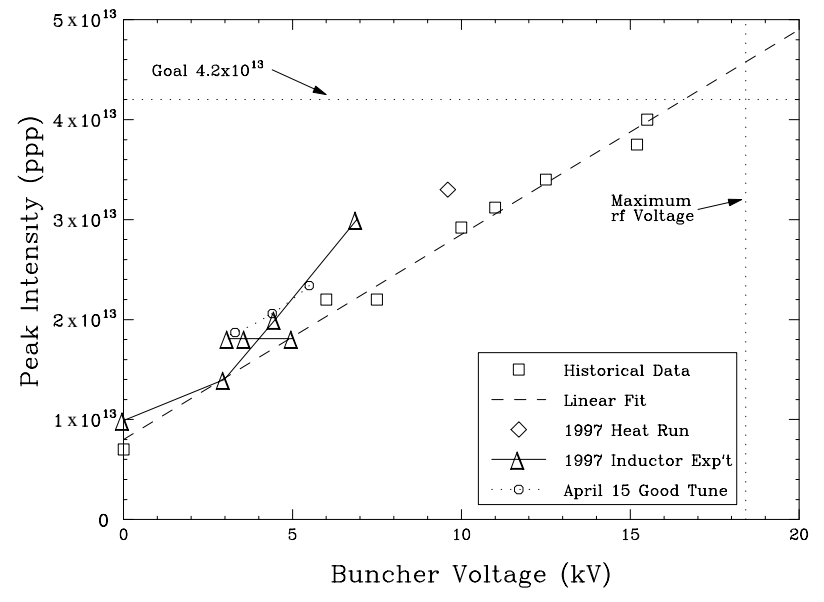

FIG. 5. Stability threshold versus rf-voltage improvement. Results of this experiment are depicted by triangles.

needed. This observation should be tempered by noting that the solenoidal field introduces extra complications that may tend to lower the threshold. In fact, as the solenoidal field was ramping-up to its set point, the general character of the measurement of beam loss as a function of time changed. During the current ramp, the beam appeared to go from slightly unstable, to stable, to very unstable, to stable. The time distribution of losses also changed. The observation of changes in losses and stability during the solenoid ramp was made at only one intensity, and because no carefully recorded measurements were made of this phenomenon, it should be regarded as a casual observation. It has been suggested that solenoidal bias windings be wrapped on some of the PSR straight-section beam pipes in an attempt to affect electron motion in the ring. Previous to this experiment, this had never been done. This may be a serendipitous result of this experiment. It has further been suggested [31] that even though linear solenoid focusing effects are insignificant, the solenoid also generates small nonlinear (octupole-like) focusing effects, which may affect the beam stability. Nonlinear focusing fields increase the betatron tune spread slightly, thereby contributing stabilizing Landau damping.

A second series of instability measurements, also shown in Fig. 5, was made on the second day. For these measurements, the onset of large vertical oscillations, measured by a short-stripline beam-position monitor, was used to locate the threshold. For a $1025 \mu$ s beam gate, $250 \mathrm{~ns}$ pulse length, and $4.8 \mu \mathrm{C}$ of charge, or $3.0 \times 10^{13}$ protons in the ring, the beam was marginally unstable for rf voltage of $6.904 \mathrm{kV}$ (flat top). Three more data points with less charge in the ring were obtained by lowering the beam current using adjustable aperture jaws in the low-energy transport portion of the linac. Beam charge in the ring, $\mathrm{rf}$ voltage and waveform, and ferrite bias condition are listed in Table I. At the highest beam intensity the required rf voltage was reduced to $60 \%$ of that which had previously been necessary to maintain stability. 
TABLE I. Buncher voltages needed for the marginally stable beam. Note that $4.8 \mu \mathrm{C}$ corresponds to $3.0 \times 10^{13}$ protons.

\begin{tabular}{cccl}
\hline \hline No. & Beam charge $(\mu \mathrm{C})$ & Buncher voltage $(\mathrm{kV})$ & \multicolumn{1}{c}{ Comment } \\
\hline 1 & 2.9 & 5.0 & Ramped buncher \\
2 & 2.9 & 3.6 & Flat top buncher \\
3 & 2.9 & 3.1 & Flat top, solenoid at 900 A \\
4 & 5.0 & $<7.7$ & Flat top buncher \\
5 & 4.8 & 6.9 & Flat top buncher \\
6 & 3.2 & 4.5 & Flat top buncher \\
7 & 2.2 & 3.0 & Flat top buncher \\
8 & 1.5 & 0.0 & Buncher turned off \\
\hline \hline
\end{tabular}

From simple beam dynamics concepts, it is expected that the inductor should provide a beam intensity-dependent effect on the instability threshold, and the data suggest that this is, in fact, the case. As the beam intensity is increased, the data points with the inductor installed move farther to the left of the historical data points, as shown in Fig. 5. A clear and unambiguous indication that the inductor raises the instability threshold would be data points that lie farther to the left (toward lower rf voltage) for a given beam charge than any of the historical measurements. However, for the low-intensity data points, there exists an earlier set of measurements with the same buncher voltages (within errors) as the inductor measurements, so it cannot be stated conclusively that the inductor raises the instability threshold for all intensities.

The instability threshold without the inductor has been shown [32] to depend on the ring revolution frequency, the rf phase with respect to the injected pattern, and the transverse beam size. In general, to minimize the required rf voltage (i.e., optimize the instability threshold), the beam must be injected into the center of the rf separatrices and the beam size must be large. For measurements 1-4 in Table I, the energy and beam size were optimized but the rf phase was not. For measurements $5-8$, the rf phase was empirically optimized, but for these measurements (on the second day of the experiment), there is some evidence that the linac energy may have drifted slightly. Unfortunately, there is not enough information available to correct for this effect with confidence.

\section{CONCLUSION}

The contention [9] that longitudinal space-charge fields in high-current proton rings operating below transition can be compensated for passively through the introduction of beam chamber inductance is supported by the results of this experiment and by a concurrent KEK Proton Synchrotron experiment [33] (see "Note Added"). The concern that introduction of additional inductive reactance into the vacuum chamber of such a ring will cause undesirable instabilities appears to be unwarranted. No such instabilities were observed in the LANL PSR up to intensity $3 \times 10^{13}$ protons per pulse.
These results [7,34] are sufficiently encouraging to warrant construction of additional passive inductors similar to those used in this experiment. In a storage ring such as the PSR, the gated linac beam is injected with some fixed pattern length. This causes the line charge distribution to be nearly rectangular, with short, steep, exponentially decaying edges. The longitudinal space-charge fields, being proportional to the derivative of the line charge distribution along the orbit, will have large high-frequency components at each end of the bunch. It is important to preserve the bandwidth of the compensation system to sufficiently high harmonics of the rotation frequency to effectively compensate for these high-frequency components. If this is not done, the large fields at each end of the bunch will force protons into the gap and the effectiveness of the system will be lost. The ferrite chosen seems well suited to this application. A different ferrite, with higher relative permeability, might be chosen in order to obtain the required inductance with a shorter structure. However, this would cause the bandwidth over which the inductor retains its value to be compromised. Problems resulting from the time limitation placed on application of the ferrite bias during the experiment can be resolved by design of water-cooled bias windings. The bunch-shape effects predicted and observed are not dramatic, but the results show that an inductor of about $8 \mu \mathrm{H}$ could significantly reduce the required buncher voltages. For example, at a beam intensity of $4.8 \mu \mathrm{C}$ a reduction of $37 \%$ was observed. As has been pointed out, in a sinusoidally rf-generated phase-space contour (bucket), bunch length scales with rf voltage as $V_{\mathrm{rf}}^{-1 / 4}$. Relatively small decreases in bunch length obtained by cancellation of space-charge forces through introduction of inductance can be equivalent to substantially larger and more costly increases in rf voltage.

\section{ACKNOWLEDGMENTS}

The Fermi National Accelerator Laboratory is operated by the Universities Research Association, Inc., under contract with the U.S. Department of Energy.

Note Added. - In early 1997, a related experiment was started at the KEK Proton Synchrotron main ring [33] in 
Japan. At injection energy, $500 \mathrm{MeV}$, the space-charge impedance of that machine is $\sim 440 \Omega$. An impedance consisting of eight FINEMET cores was installed in the ring. (FINEMET is a very high relative permeability material, which is also very lossy at $\mathrm{MHz}$ frequencies. The $Q$ of the inserted cores was less than one over the frequency range of the experiment.) The rings were encircled by a figure-eight bias winding coil so that the inserted inductance could be reduced substantially under controlled conditions. In the experiment, the incoherent synchrotron phase oscillation frequency, inferred by measurement of the quadrupole bunch motion frequency, was measured as a function of beam intensity. Between $3 \times 10^{11}$ and $9 \times 10^{11}$ protons per bunch, the slope of the incoherent frequency vs intensity was reduced by half over the same measurement with no compensating inductance. This result is consistent with the predicted result since only half the required compensation inductance was placed in the ring. No deleterious effects have been reported.

A salient point regarding this result is that it indicates clearly that $\mathrm{rf}$ components of beam image current and the associated electric and magnetic fields can link lumped inductances introduced into the vacuum chamber in such a way as to generate effective longitudinal electric fields.

\section{APPENDIX: INDUCTOR CHARACTERIZATION}

\section{A. Permeability measurements on the ferrite toroids}

In July 1997, a set of inductance measurements was made at LANL on one of the ferrite rings, using an $L-C$ oscillation method. The inductor was formed by the ring in a single-turn housing and the capacitor by discrete capacitors. The test fixture was made by cutting and soldering copper foil to make a closely fitting toroidal housing. Some effort was made to minimize stray inductances, but there apparently was some residual stray inductance in series with the inductor formed by the toroid and housing since measured inductances were consistently higher than the manufacturer's. High-voltage low-loss plastic capacitors mounted on the top plate were used for the capacitive element. Since $Q$ 's for the ringing were on the order of 10 or 20 and the manufacturer's $Q$ was 100 for frequencies less than $20 \mathrm{MHz}$, there apparently were copper losses in the leads that lowered the measured $Q$.

After the capacitors were charged with a dc supply (the largest voltage used was $240 \mathrm{~V}$ ); a mercury-wetted relay was closed to initiate oscillations. A high-voltage probe and digital oscilloscope were used to record the voltage across the inductor as a function of time. The traces were sinusoidal with an exponentially decaying envelope. Oscillation period data were obtained from these traces and used, together with the capacitance values, to compute inductances and permeabilities. To determine the linearity of the ferrite for each capacitance value, various charging voltages between $120 \mathrm{~V}$ and $240 \mathrm{~V}$ were used and the oscillation frequencies measured. These charging voltages exceed the voltage drop that occurred across a single toroid of the inductor stack in the PSR. The resultant flux densities also exceed those encountered in the PSR. Also, there was no change observed in the permeability within the experimental error as the voltage was varied at a given frequency. In conclusion, these results show that the ferrite material is very linear over the range of flux densities encountered in the PSR.

\section{B. Inductance measurements}

After removing the inductor from the PSR, a series of low-frequency $(0.5-14 \mathrm{MHz})$ inductance measurements [35] was made on the inductor at the same set of biascurrent levels that were used in the PSR experiments.

The impedance of the inductor was measured with a Hewlett-Packard 8751 Network Analyzer in a singleport mode. The center conductor of a short $50 \Omega$ cable from the measuring port of the network analyzer was connected to the aluminum rod, and the cable sheath was connected to a brass plate on the near end of the inductor, as shown in Fig. 6. The aluminum rod was shorted to a brass plate on the other end of the inductor. The contribution of the cable to the impedance was calibrated out of the measurement. Cable currents involved in the measurement were not measured but were small compared with the beam currents in the PSR experiment. The impedance measured in this setup is basically that of a shorted ferrite-loaded transmission line (TEM mode). The lowest-frequency resonance in this case is a quarter-wave resonance with a frequency of about $25 \mathrm{MHz}$ with zero ferrite bias (for each of the two cavities alone) and with a higher resonant frequency with increasing ferrite bias field. Resonant effects in the PSR experiment involve different boundary conditions since when the inductor is in the ring there is no center conductor. The lowest-frequency mode with the beamdriven inductor is a $\mathrm{TM}_{010}$ mode with a considerably higher resonant frequency. The most that can be expected from the network analyzer measurement is therefore the dependence of the low-frequency inductance on bias current.

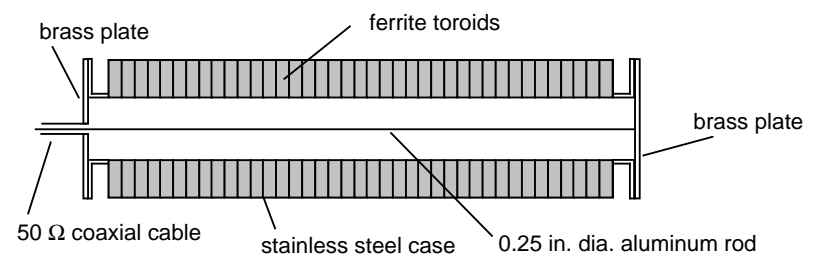

FIG. 6. Schematic cross section of the measurement setup with test fixtures. 


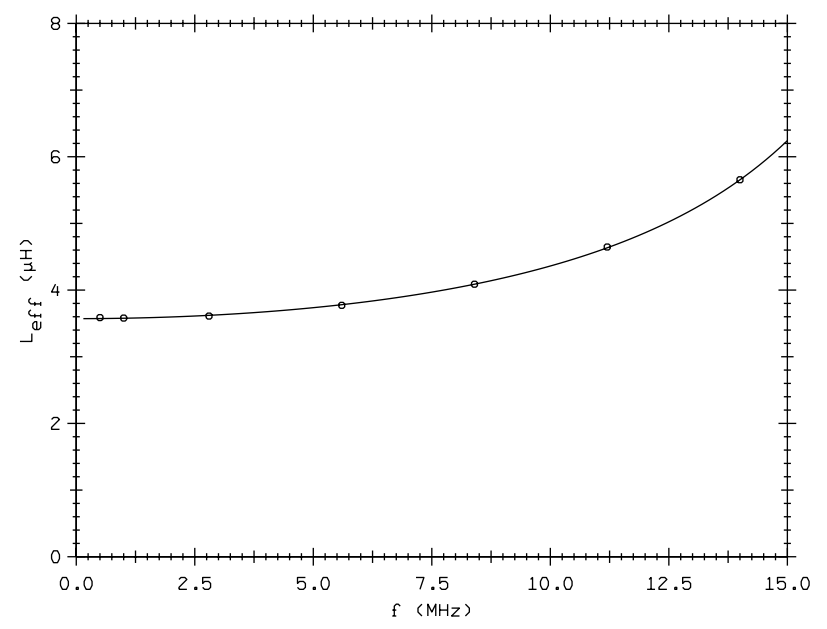

FIG. 7. Dependence of effective inductance on frequency for one inductive cavity with zero bias current. Measured data are indicated by circles. The solid curve is a fit to the data using a TEM-mode resonance expression.

\section{Results}

The dependence of the effective inductance on frequency for a single cavity with zero bias current is shown in Fig. 7. The shorted transmission-line formula for the impedance at frequency $f$ is

$$
Z=j Z_{c} \tan \left(\frac{\pi f}{2 f_{\text {res }}}\right) .
$$

The effective inductance is defined to be

$$
L_{\text {eff }}=\frac{\operatorname{Re} Z_{c}}{2 \pi f} \tan \left(\frac{\pi f}{2 f_{\text {res }}}\right) .
$$

In the two-parameter fit of Fig. 7, $f_{\text {res }}$ is $21.7 \mathrm{MHz}$ and $\operatorname{Re} Z_{c}$ is $310 \Omega$. If the losses are due to ferrite hysteresis, they can be roughly modeled by allowing the characteristic impedance $Z_{c}$ to have a negative imaginary part.

Figure 8 is a fit to the low-frequency points of the real part of the impedance data with $\operatorname{Im} Z_{0}=-2.2 \Omega$ and $f_{\text {res }}=21.7 \mathrm{MHz}$. From the figure, it is clear that there must be contributions to the real part of the impedance at high frequency in addition to the those modeled by Eq. (A1) with a constant imaginary part to $Z_{c}$. Since part of the losses may be frequency-dependent artifacts of the

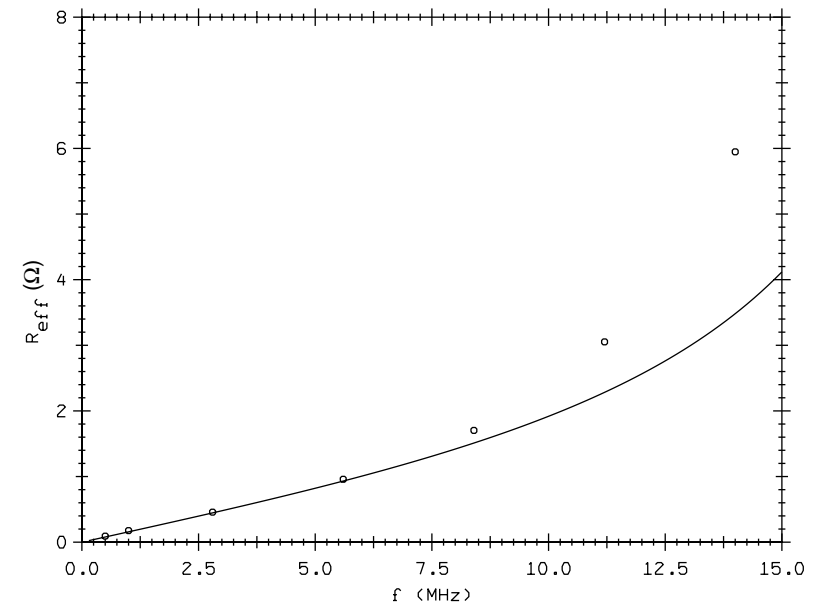

FIG. 8. Dependence of the real part of the impedance on frequency for one cavity with zero bias current. The solid curve is a fit to the lowest-frequency points assuming a constant negative imaginary part to the characteristic transmission-line impedance.

measurement setup (e.g., eddy currents in the aluminum rod, etc.), no definite conclusion regarding ferrite losses can be made from these data alone.

The measured low-frequency effective inductance is listed in Table II for the various bias currents. This inductance can be obtained by extrapolating fits like those of Fig. 7 to zero frequency or more simply by taking the measured values at $500 \mathrm{kHz}$, which is a frequency low enough to ensure that the resonance does not affect the value. The summed inductance data of Table II are plotted in Fig. 9.

Impedance measurements were also made with the two cavities together, with an aluminum rod that passed through both cavities and was shorted at the end of the second cavity. As expected, the resonance frequency of the two cavities together was lower than that for the individual cavities; the resonance was at $12.5 \mathrm{MHz}$ for the combined cavities. Low-frequency inductances for the combined cavities agreed to better than 5\% with the summed inductances of the separate cavities. However, since the resonance effects are less pronounced for the separate cavities, the summed inductances of the separate cavities were taken for the best values of the inductance.

TABLE II. Measured low-frequency inductances.

\begin{tabular}{cccc}
\hline \hline Bias current (A) & $\begin{array}{c}\text { Inductance of } \\
\text { cavity 1 }(\mu \mathrm{H})\end{array}$ & $\begin{array}{c}\text { Inductance of } \\
\text { cavity 2 }(\mu \mathrm{H})\end{array}$ & $\begin{array}{c}\text { Sum of inductances } \\
\text { cavities 1 and 2 }(\mu \mathrm{H})\end{array}$ \\
\hline 0 & 3.59 & 3.70 & 7.29 \\
200 & 2.42 & 2.38 & 4.81 \\
600 & 1.45 & 1.46 & 2.92 \\
900 & 1.24 & 1.25 & 2.49 \\
1000 & 1.20 & 1.20 & 2.40 \\
\hline \hline
\end{tabular}




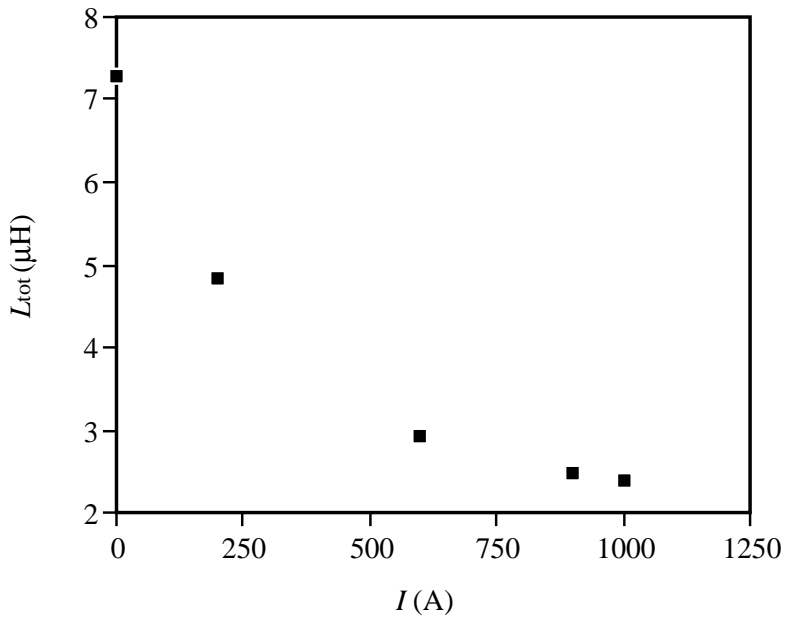

FIG. 9. Dependence of summed inductance of the two cavities on bias current.

\section{Discussion}

Results of calculations with the computer program MAFIA are shown in Fig. 10. These calculations, which assume a relative permeability of 70 and a relative dielectric constant of 10 for the ferrite, predict an inductance of $8.8 \mu \mathrm{H}$ at $2.795 \mathrm{MHz}$, which is close to the measured value of $7.29 \mu \mathrm{H}$. The calculation also predicts a resonant frequency of $75 \mathrm{MHz}$ for a beam-driven cavity (no center conductor, $\mathrm{TM}_{010}$ mode), but the actual resonant frequency is probably higher because of the falloff of the permeability at high frequency. The manufacturer's data for $\mathrm{M}_{4} \mathrm{C}_{21 \mathrm{~A}}$ show that the permeability is constant with frequency out to about $30 \mathrm{MHz}$ and then falls off sharply. Similarly, according to the manufacturer, the quality factor is constant with frequency at a value of about 150 and falls off at about the same frequency. Measurement with single toroids of the same lot as those used in the inductor demonstrated the linearity of the ferrite in the 1 to $10 \mathrm{MHz}$ range (i.e., lack of dependence of the permeability on the ac current in the beam or center conductor). This linearity implies

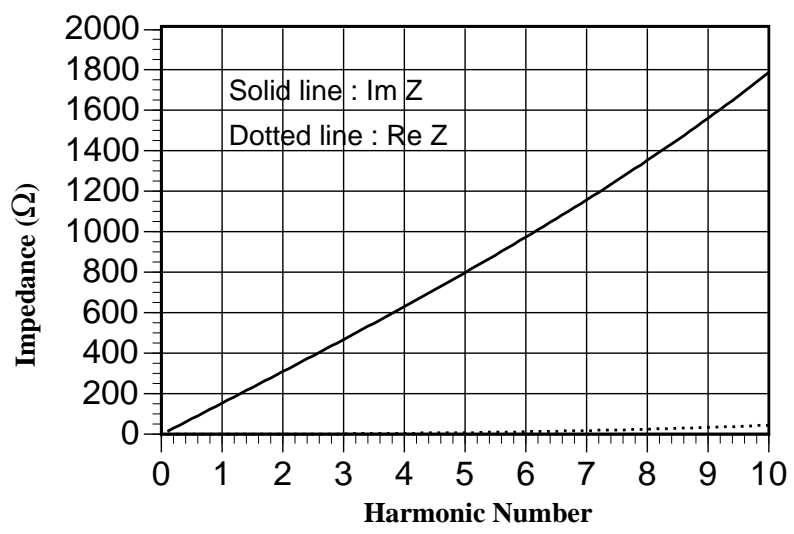

FIG. 10. Inductor module impedance (total of both cavities) from MAFIA calculations. that low-current inductance measurements should give the same inductances as those "seen" by the beam, at least for moderate frequencies ( 0 to $20 \mathrm{MHz}$ ). For modeling purposes, therefore, the best procedure seems to be to use the data of Table II for the dependence of inductance on bias current, the manufacturer's data for the quality factor and dependence of permeability on frequency, and MAFIA or Superfish calculations for the resonance behavior of the cavities.

[1] J. R. Alonso, in Proceedings of the Sixth European Particle Accelerator Conference, Stockholm, Sweden, 1998, IOP Conf. Proc. (Institute of Physics and Physical Society, London, 1998), p. 493.

[2] JHF Project Office, JHF Accelerator Design Study Report, KEK Report No. 97-16, March 1998 (unpublished).

[3] "ESS, A Next Generation Neutron Source for Europe," ESS Technical Study (unpublished), Vol. 3.

[4] C. Akenbrandt et al., "A Development Plan for the Fermilab Proton Source," edited by S. Holmes, Fermilab Report TM-2021 (unpublished).

[5] V. K. Neil and A. M. Sessler, Rev. Sci. Instrum. 36, 429 (1965).

[6] S. Hansen, H. G. Hereward, A. Hofmann, K. Hübner, and S. Myers, IEEE Trans. Nucl. Sci. 22, 1381 (1975).

[7] J.E. Griffin, K.Y. Ng, Z.B. Qian, and D. Wildman, Fermilab Tech. Note FN-661, 1997 (unpublished).

[8] C. E. Nielsen, A. M. Sessler, and K. R. Symon, in Proceedings of the International Conference on High-Energy Accelerators and Instrumentation (CERN, Geneva, 1959), p. 239.

[9] A.M. Sessler and V.G. Vaccaro, CERN Report No. CERN 68-1, 1968 (unpublished).

[10] R. K. Cooper, D. W. Hudgings, and G. P. Lawrence, LANL PSR Tech. Note 100, 1982 (unpublished).

[11] T. Hardek, LANL PSR Tech. Note 102, 1982 (unpublished).

[12] V. K. Neil and R. J. Briggs, Plasma Phys. 9, 631 (1967).

[13] R. J. Briggs and V. K. Neil, J. Nucl. Energy, Part C 8, 255 (1966).

[14] D. Neuffer et al., Part. Accel. 23, 133 (1988).

[15] E. Colton et al., Proceedings of the IEEE Particle Accelerator Conference, San Francisco, CA, 1991 (IEEE, New York, 1991), p. 1896.

[16] D. Neuffer et al., Nucl. Instrum. Methods Phys. Res., Sect. A 321, 1 (1992).

[17] T.S. Wang, Proceedings of the 1995 Particle Accelerator Conference and the International Conference on High Energy Accelerators, edited by L. Gennari (IEEE, Piscataway, NJ, 1996), p. 3134.

[18] M. Plum et al., Proc. 1995 Part. Accel. Conf. and Int. Conf. High Energy Accel. (Ref. [17]), p. 3406.

[19] R. Macek and H. A. Thiessen, SPSS Accelerator Enhancement Project, Technical Overview, WBS 1.1.0, 1997 (unpublished).

[20] J.E. Griffin, C. Ankenbrandt, J.A. MacLachlan, and A. Moretti, IEEE Trans. Nucl. Sci. 30, 3502 (1983); V. K. Bharadwaj, J.E. Griffin, D. J. Harding, and J. A. MacLachlan, IEEE Trans. Nucl. Sci. 34, 1025 (1987). 
[21] J.E. Griffin, Status Review of LANL PSR Performance Upgrade Plans, March 1997 (unpublished).

[22] E. Keil and W. Schnell, CERN Report No. CERN-ISRTH-RF/69-48, 1969 (unpublished).

[23] P. Channell and T. S. Wang, LANL Report No. LA-UR96-1844, 1996 (unpublished).

[24] E. Colton (private communication).

[25] K. Y. Ng, Phys. Rev. D 42, 1819 (1990).

[26] K. Y. Ng and Z. Qian, Fermilab Tech. Note FN-659, 1997 (unpublished); Workshop on Physics at the First Muon Collider and at the Front End of the Muon Collider, edited by S. Geer and R. Raja, AIP Conf. Proc. No. 435 (AIP, New York, 1998), p. 841.

[27] K. Y. Ng and Z. Qian (unpublished).
[28] P. L. Walstrom (private communication).

[29] J. E. Griffin, P. D. Coleman, and J. Langstrand, Fermilab Tech. Note TM-2038, 1997 (to be published).

[30] D. Fitzgerald, LANL PSR Tech. Note PSR-95-010, 1995 (unpublished).

[31] S. Nagaitsev (private communication).

[32] M. A. Plum et al., LANL PSR Tech. Note PSR-97-019, 1997 (unpublished).

[33] K. Koba, S. Machida, and Y. Mori, KEK JHF-Ring Memo 309, 1997 (unpublished).

[34] M. A. Plum et al., LANL PSR Tech. Note PSR-98-013, 1998 (unpublished).

[35] P. L. Walstrom, LANL PSR Tech. Note PSR-98-03, 1998 (unpublished). 